Springer Link

\title{
Moisture Control Capacity of Geopolymer Composites: Correlation of the Bulk Composition- Pore Network with the Absorption-Desorption Behavior
}

\author{
Transport in Porous Media \\ March 2018, Volume 122, Issue 1, pp 77-95 | Cite as
}

- E. Kamseu (1) (2) Email author (kamseuelie@yahoo.fr)

- H. Mohamed (2)

- J. C. Sofack (2)

- Duangrudee Chaysuwan (3)

- H. K. Tchakoute (4)

- J. N. Y. Djobo (2)

- S. Rossignol (5)

- C. Leonelli (1)

1. Department of Engineering "Enzo Ferrari”, University of Modena and Reggio Emilia, , Modena, Italy 2. Local Materials Promotion Authority/MIPROMALO, , Nkolkok Yaoundé, Cameroon

3. Department of Materials Engineering, Faculty of Engineering, Kasetsart University, , Bangkok, Thailand

4. Laboratory of Applied Inorganic Chemistry, Faculty of Science, University of Yaoundé I, , Yaoundé,

Cameroon

5. European Ceramic Centre, SCPTS, UMR CNRS 7315, , Limoges Cedex, France

Article

First Online: 28 December 2017

- 384 Downloads

\begin{abstract}
Porous composites with the principal class of porosity in the range of those presented in the literature as ideal for the moisture control capacity of building environment are described. In the course of the design of the matrices, micrometric pores are introduced to give to the pore systems a bi- or multimodal characters with the aim of improving the phases percolation during the course of desorption and make the moisture accumulation-desorption behavior of the porous composites essentially function of weather and environment. The porous composites present size of pores in the range $0.001-1 \mu \mathrm{m}$ for the gel pores and peak centered at $10 \mu \mathrm{m}$ for the micrometric pores which insure the matrices efficiency in moisture control capacity and durability. The results of cycles of moisture absorption-desorption in the course of various seasons of the year permit to identify the activities of gel pores meanly efficient in the extreme environment: absorption when the temperature is under $11^{\circ} \mathrm{C}$; relative humidity is $>60 \%$ and desorption when the temperature is above $18^{\circ} \mathrm{C}$. At ambient conditions, the pores more active are micrometric pores, while gel pores enter in activity only in the extreme environment conditions. The proposed porous geopolymer composites appeared promising candidates for the management of the moisture while improving the thermal insulation of residential building particularly in the regions with important fluctuation of weather. The use of geopolymerization process for the production of those porous composites, the choice of recycling industrial and municipal inorganic wastes appears ideal solution, environmentally friendly, eco-efficient and sustainable for the design of newly materials for the moisture control capacity in building environment.
\end{abstract}

\section{Graphical Abstract}




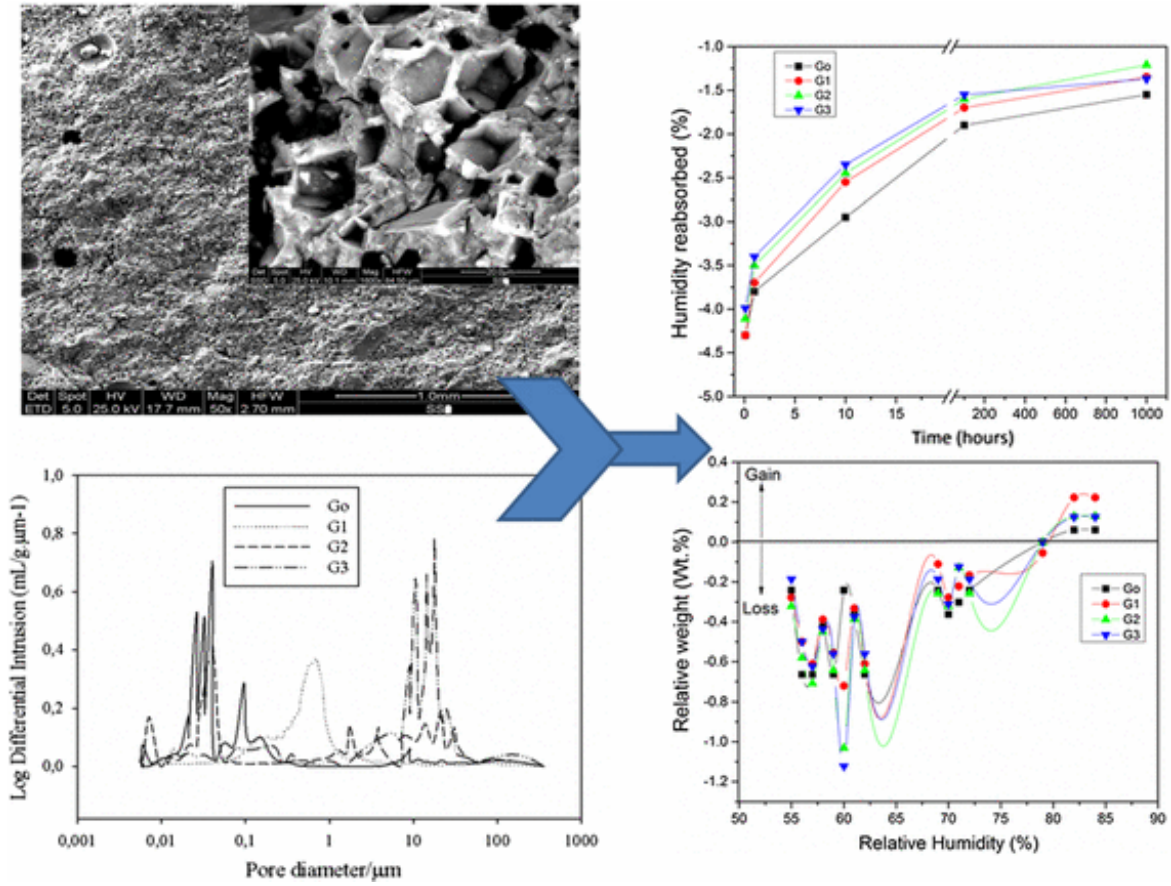

\section{Keywords}

Porous geopolymer composites Gel pores Absorption-desorption Moisture control capacity This is a preview of subscription content, $\underline{\log } \underline{\text { in }}$ to check access.

\section{Notes}

\section{Acknowledgements}

We acknowledge the laboratory Ingessil Srl Verona for providing the sodium silicate used for the samples preparation. The research grants received from Universitary Agency of Francophony (AUF) and the Academy of Science for the third world (TWAS) to the principal investigator Dr. Elie Kamseu are also acknowledged.

\section{Funding}

Funding was provided by Academy of Science for the Third World TWAS (Grant No. AF/AC-I-FR 3240287064).

\section{References}

Alonso, S., Palomo, A.: Alkaline activation of metakaolin-calcium hydroxide solid mixtures: influence of temperature, activator concentration and metakaolin $/ \mathrm{Ca}(\mathrm{OH}) 2$ ratio. Mater. Lett. 47, 55-62 (2001)

CrossRef (https://doi.org/10.1016/So167-577X(00)00212-3)

Google Scholar (http://scholar.google.com/scholar_lookup?

title=Alkaline\%20activation\%20of\%2ometakaolin\%E2\%80\%93calcium\%2ohydroxide\%2osolid\%2omixture s\%3A\%2oinfluence\%20of\%2otemperature\%2C\%20activator\%2oconcentration\%2oand\%2ometakaolin\%2F Ca\%28OH\%292\%2oratio\&author=S.\%20Alonso\&author=A.\%2OPalomo\&journal=Mater.\%2OLett.\&volume $=47 \&$ pages $=55-62 \&$ publication_year $=2001$ )

Alonso, S., Palomo, A.: Calorimetric study of alkaline activation of calcium hydroxide-metakaolin solidmixtures. Cem. Concr. Res. 31, 25-30 (2001)

CrossRef (https://doi.org/10.1016/Sooo8-8846(00)o0435-X)

Google Scholar (http://scholar.google.com/scholar_lookup?

title=Calorimetric\%2ostudy\%20of\%20alkaline\%20activation\%20of\%2ocalcium\%2ohydroxide\%E2\%80\%93 
metakaolin\%20solid-

mixtures\&author=S.\%20Alonso\&author=A.\%20Palomo\&journal=Cem.\%20Concr.\%20Res.\&volume=31\&pa ges=25-30\&publication_year $=2001$ )

Belitsky, I.V., Sakata, A., Goto, S.: Kinetics of hydration of slag in the slag-alkaline cements. In: Proceedings of the 3rd Beijing International Symposium on Cement and Concrete, Beijing, China, 27-30 Oct 1993, pp. 1028-1031. International Academic Publishers, San Bernardino, CA (1993)

Google Scholar (https://scholar.google.com/scholar?

q=Belitsky\%2C\%2OI.V.\%2C\%20Sakata\%2C\%20A.\%2C\%20Goto\%2C\%20S.\%3A\%2oKinetics\%2oof\%2ohydr ation\%20of\%20slag\%20in\%20the\%20slag-

alkaline\%20cements.\%20In\%3A\%20Proceedings\%20of\%20the\%203rd\%20Beijing\%2oInternational\%20Sy mposium\%20on\%20Cement\%2oand\%2oConcrete\%2C\%2oBeijing\%2C\%20China\%2C\%2027\%E2\%80\%933 o\%20Oct\%201993\%2C\%2Opp.\%201028\%E2\%80\%931031.\%2oInternational\%2oAcademic\%2oPublishers\% 2C\%2oSan\%2oBernardino\%2C\%20CA\%20\%281993\%29)

Bignozzi, M.C., Manzi, S., Lancellotti, I., Kamseu, E., Barbieri, L., Leonelli, C.: Mix-design and characterization of alkali activated materials based on metakaolin and ladle slag. Appl. Clay Sci. 73, 78-85 (2013)

CrossRef (https://doi.org/10.1016/j.clay.2012.09.015)

Google Scholar (http://scholar.google.com/scholar_lookup?title=Mix-

design\%20and\%20characterization\%20of\%20alkali\%20activated\%2omaterials\%2obased\%20on\%2ometak aolin\%20and\%2oladle\%2oslag\&author=MC.\%2oBignozzi\&author=S.\%20Manzi\&author=I.\%2oLancellotti \&author=E.\%20Kamseu\&author=L.\%2OBarbieri\&author=C.\%2OLeonelli\&journal=Appl.\%20Clay\%20Sci.\& volume $=73$ \&pages $=78-85$ \&publication_year $=2013$ )

Carsten, C., Peuhkuri, R., Time, B., Svennberg, K., Ojanen, T.: Moisture buffer value of building materials. In: ASTM Symposium on Heat-Air-Moisture Transport: Measurement on Building Materials, Toronto, 23 April (2006)

Google Scholar (https://scholar.google.com/scholar?

q=Carsten\%2C \% 20C.\%2C\%2OPeuhkuri\%2C\%20R.\%2C\%2OTime\%2C\%2oB.\%2C\%2oSvennberg\%2C\%2OK. \%2C\%2oOjanen\%2C\%20T.\%3A\%20Moisture\%2obuffer\%20value\%2oof\%2obuilding\%2omaterials.\%2oIn \%3A\%20ASTM\%20Symposium\%20on\%20Heat\%E2\%80\%93Air\%E2\%80\%93Moisture\%20Transport\%3A \%20Measurement\%20on\%2oBuilding\%2oMaterials\%2C\%2OToronto\%2C\%2023\%20April\%20\%282006\%2 9)

Gezer, N. A.: The effects of construction materials on thermal comfort in residential buildings, an analysis using Ecotect 5.o. Thesis, Middle East Technical University (2003)

Google Scholar (https://scholar.google.com/scholar? q=Gezer\%2C\%2oN.\%20A.\%3A\%20The\%2oeffects\%20of\%2oconstruction\%2omaterials\%20on\%20thermal \%20comfort\%20in\%2oresidential\%2obuildings\%2C\%20an\%20analysis\%20using\%2oEcotect\%205.0.\%20T hesis\%2C\%20Middle\%2oEast\%20Technical\%2oUniversity\%20\%282003\%29)

Kamseu, E., Nait-Ali, B., Bignozzi, M.C., Leonelli, C., Rossignol, S., Smith, D.S.: Bulk composition and microstructure dependence of effective thermal conductivity of porous inorganic polymer cements. J. Eur. Ceram. Soc. 32(8), 1593-1603 (2012)

CrossRef (https://doi.org/10.1016/j.jeurceramsoc.2011.12.030)

Google Scholar (http://scholar.google.com/scholar_lookup?

title=Bulk\%20composition\%20and\%20microstructure\%2odependence\%20of\%2oeffective\%2othermal\%20 conductivity\%20of\%2oporous\%20inorganic\%20polymer\%20cements\&author=E.\%20Kamseu\&author=B.\% 20Nait-

Ali\&author=MC.\%2oBignozzi\&author=C.\%2oLeonelli\&author=S.\%20Rossignol\&author=DS.\%20Smith\&jo urnal=J.\%20Eur.\%20Ceram.\%20Soc. \&volume=32\&issue=8\&pages=1593-1603\&publication_year=2012)

Kamseu, E., Cannio, M., Obonyo, E.A., Tobias, F., Bignozzi, M.C., Sglavo, V.M., Leonelli, C.: Metakaolinbased inorganic polymer composite: effects of fine aggregate composition and structure on porosity evolution, microstructure and mechanical properties. Cem. Concr. Compos. 53, 258-269 (2014)

CrossRef (https://doi.org/10.1016/j.cemconcomp.2014.07.008)

Google Scholar (http://scholar.google.com/scholar_lookup?title=Metakaolin-

based\%20inorganic\%20polymer\%20composite\%3A\%20effects\%20of\%2ofine\%20aggregate\%20compositio n\%20and\%20structure\%20on\%2oporosity\%2oevolution\%2C\%2omicrostructure\%20and\%2omechanical\%2 oproperties\&author=E.\%20Kamseu\&author=M.\%20Cannio\&author=EA.\%20Obonyo\&author=F.\%20Tobia s\&author=MC.\%20Bignozzi\&author=VM.\%20Sglavo\&author=C.\%2OLeonelli\&journal=Cem.\%2oConcr.\%2 oCompos.\&volume $=53 \&$ pages $=258-269 \&$ publication_year $=2014)$

Kamseu, E., Ponzoni, C., Tippayasam, C., Taurino, R., Chaysuwan, D., Bignozzi, M.C., Barbieri, L., Leonelli, C.: Influence of fine aggregates on the microstructure, porosity and chemico-mechanical stability of inorganic polymer concretes. Constr. Build. Mater. 96, 473-483 (2015)

CrossRef (https://doi.org/10.1016/j.conbuildmat.2015.08.090)

Google Scholar (http://scholar.google.com/scholar_lookup?

title=Influence $\%$ 20of\%2ofine\%20aggregates $\% 200$ \% $\%$ 20the\%2omicrostructure $\% 2 \mathrm{C} \%$ 20porosity $\% 20$ and $\% 2$ ochemico- 
mechanical\%20stability\%20of\%20inorganic\%20polymer\%20concretes\&author=E.\%20Kamseu\&author=C. \%20Ponzoni\&author=C.\%20Tippayasam\&author=R.\%20Taurino\&author=D.\%20Chaysuwan\&author=MC. \%20Bignozzi\&author=L.\%2oBarbieri\&author=C.\%20Leonelli\&journal=Constr.\%2oBuild.\%2oMater.\&volu me=96\&pages $=473-483 \&$ publication_year $=2015$ )

Kamseu, E., NGouloure, Z.N.M., Nait Ali, B., Zekeng, S., Melo, U.C., Rossignol, S., Leonelli, C.: Cumulative pore volume, pore size distribution and phases percolation in porous inorganic polymer composites: relation microstructure and effective thermal conductivity. Energy Build. 88, 45-56 (2015)

CrossRef (https://doi.org/10.1016/j.enbuild.2014.11.066)

Google Scholar (http://scholar.google.com/scholar_lookup?

title=Cumulative\%2opore\%20volume\%2C\%20pore\%20size\%20distribution\%20and\%2ophases\%2opercolat ion\%20in\%2oporous\%2oinorganic\%20polymer\%20composites\%3A\%2orelation\%2omicrostructure\%20an d\%20effective\%2othermal\%20conductivity\&author=E.\%20Kamseu\&author=ZNM.\%20NGouloure\&author =B.\%20Nait\%20Ali\&author=S.\%20Zekeng\&author=UC.\%20Melo\&author=S.\%2oRossignol\&author=C.\%2 oLeonelli\&journal=Energy\%2oBuild.\&volume=88\&pages=45-56\&publication_year=2015)

Kamseu, E., Lancelotti, I., Sglavo, V.M., Modolo, I., Leonelli, C.: Design of inorganic polymer mortar from ferricalsialic and calsialic slags for indoor humidity control. Materials (2016).

https://doi.org/10.3390/ma9060410 (https://doi.org/10.3390/ma9060410)

Google Scholar (http://scholar.google.com/scholar_lookup?

title=Design\%20of\%2oinorganic\%2opolymer\%2omortar\%2ofrom\%2oferricalsialic\%2oand\%2ocalsialic\%2 oslags\%2ofor\%2oindoor\%20humidity\%20control\&author=E.\%20Kamseu\&author=I.\%2oLancelotti\&autho $\mathrm{r}=\mathrm{VM} . \% 20$ Sglavo\&author=I.\%20Modolo\&author=C.\%20Leonelli\&journal=Materials\&publication_year=20 16\&doi=10.3390\%2Fma9060410)

Kamseu, E., Ponzoni, C., Tippayasam, C., Taurino, R., Chaysuwan, D., Sglavo, V.M., Thavorniti, P., Leonelli, C.: Self-compacting geopolymer concretes: effects of addition of aluminosilicate-rich fines. J. Build. Eng. 5, 211-221 (2016)

CrossRef (https://doi.org/10.1016/j.jobe.2016.01.004)

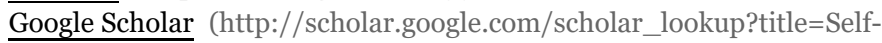
compacting\%20 geopolymer\%20concretes\%3A\%20effects\%20of\%20addition\%20of\%20aluminosilicaterich\%2ofines\&author=E.\%20Kamseu\&author=C.\%20Ponzoni\&author=C.\%20Tippayasam\&author=R.\%20 Taurino\&author=D.\%20Chaysuwan\&author=VM.\%20Sglavo\&author=P.\%20Thavorniti\&author=C.\%20Leo nelli\&journal=J.\%20Build.\%20Eng.\&volume $=5$ \&pages $=211-221 \&$ publication_year $=2016$ )

Kotama, M., Fukumizu, H., Matsumoto, Y., Tapama, M., Yamanioto, K., Endo, M.: Moisture control capacity. Construction Material USA Patent No. 64720610 B2, 12 (2002)

Google Scholar (https://scholar.google.com/scholar? q=Kotama\%2C\%2oM.\%2C\%2oFukumizu\%2C\%2OH.\%2C\%2oMatsumoto\%2C\%2OY.\%2C\%2oTapama\%2C \%20M.\%2C\%2oYamanioto\%2C\%20K.\%2C\%2oEndo\%2C\%2oM.\%3A\%20Moisture\%2ocontrol\%20capacity. \%2oConstruction\%20Material\%2oUSA\%2oPatent\%20No.\%206472\%200610\%20B2\%2C\%2012\%20\%2820 $02 \% 29)$

Kourounis, S., Tsivillis, S., Tsakiridis, P.E., Papadimitriou, G.D., Tsibouki, Z.: Properties and hydratation of blended cements with steel making slag. Cem. Concr. Res. 37, 815822 (2007)

CrossRef (https://doi.org/10.1016/j.cemconres.2007.03.008)

Google Scholar (http://scholar.google.com/scholar_lookup?

title=Properties\%20and\%2ohydratation\%20of\%2oblended\%20cements\%20with\%20steel\%2omaking\%2osl ag\&author=S.\%20Kourounis\&author $=$ S.\%20Tsivillis\&author=PE.\%20Tsakiridis\&author=GD.\%20Papadimi triou\&author=Z.\%20Tsibouki\&journal=Cem.\%20Concr.\%20Res.\&volume=37\&pages $=815822 \&$ publication _year=2007)

Kumar, A., Singh, O.P.: Advances in the building materials for thermal comfort and energy saving. J. Recent Pat. Eng. 7.3, 220-232 (2013)

CrossRef (https://doi.org/10.2174/18722121113076660005)

Google Scholar (http://scholar.google.com/scholar_lookup?

title=Advances\%2oin\%2othe\%2obuilding\%2omaterials\%2ofor\%2othermal\%20comfort\%2oand\%2oenergy \%20saving\&author=A.\%20Kumar\&author=OP.\%20Singh\&journal=J.\%20Recent\%20Pat.\%20Eng.\&volume $=7.3 \&$ pages $=220-232 \&$ publication_year $=2013$ )

Lee, W.K.W., van Deventer, J.S.J.: Chemical interactions between siliceous aggregates and low-Ca alkaliactivated cements. Cem. Concr. Res. 37, 844-55 (2007)

CrossRef (https://doi.org/10.1016/j.cemconres.2007.03.012)

Google Scholar (http://scholar.google.com/scholar_lookup?

title=Chemical\%2ointeractions\%2obetween\%2osiliceous\%2oaggregates\%20and\%2olow-Ca\%2oalkaliactivated\%20cements\&author=WKW.\%20Lee\&author=JSJ.\%2oDeventer\&journal=Cem.\%20Concr.\%20Re s.\&volume $=37 \&$ pages $=844-55 \&$ publication_year $=2007$ )

Malolepszy, J.: Some aspects of Alkali-activated cementitious materials setting and hardening. In:

Proceedings of the 3rd Beijing International Symposium on Cement and Concrete, Beijing, China, 27-30 Oct 1993, pp. 1043-1046; International Academic Publishers, San Bernardino, CA (1993) 
Google Scholar (https://scholar.google.com/scholar?

q=Malolepszy\%2C\%20J.\%3A\%20Some\%20aspects\%20of\%20Alkali-

activated\%20cementitious\%2omaterials\%20setting\%20and\%2ohardening.\%20In\%3A\%2oProceedings\%20 of\%2othe\%203rd\%2oBeijing\%2oInternational\%20Symposium\%20on\%20Cement\%2oand\%20Concrete\%2 C\%2oBeijing\%2C\%20China\%2C\%2O27\%E2\%80\%9330\%20Oct\%201993\%2C\%2Opp.\%201043\%E2\%80\%93 1046\%3B\%2oInternational\%2oAcademic\%2oPublishers\%2C\%2oSan\%2oBernardino\%2C\%20CA\%20\%281 993\%29)

Mozgawa, W., Deja, J.: Spectroscopic studies of alkaline activated slag geopolymers. J. Mol. Struct. 924-

926, $434-441$ (2009)

CrossRef (https://doi.org/10.1016/j.molstruc.2008.12.026)

Google Scholar (http://scholar.google.com/scholar_lookup?

title=Spectroscopic\%2ostudies\%200f\%20alkaline\%20activated\%20slag\%2ogeopolymers\&author=W.\%20M ozgawa\&author=J.\%20Deja\&journal=J.\%20Mol.\%20Struct.\&volume=924\%E2\%80\%93926\&pages=434441\&publication_year=2009)

Myers, R.J., Bernal, S.A., Gehman, J.D., van Deventer, J.S.J., Provis, J.L.: The role of Al in cross-linking of alkali-activated slag cements. J. Am. Ceram. Soc. 98, 996-1004 (2015)

CrossRef (https://doi.org/10.1111/jace.13360)

Google Scholar (http://scholar.google.com/scholar_lookup?

title=The\%20role\%20of\%20Al\%20in\%20cross-linking\%20of\%20alkali-

activated\%20slag\%20cements\&author=RJ.\%20Myers\&author=SA.\%20Bernal\&author=JD.\%20Gehman\&a uthor=JSJ.\%20Deventer\&author=JL.\%20Provis\&journal=J.\%20Am.\%20Ceram.\%20Soc.\&volume=98\&pag es $=996-1004 \&$ publication_year $=2015)$

Nazanin, N., Knight, I., Jones, P.: Work place satisfaction and thermal comfort in air conditioned office buildings: findings from a summer survey and field experiments in Iran. Indoor Built Environ. 17, 69-79 (2008)

CrossRef (https://doi.org/10.1177/1420326Xo7086945)

Google Scholar (http://scholar.google.com/scholar_lookup?

title=Work\%20place\%20satisfaction\%20and\%20thermal\%20comfort\%2oin\%20air\%2oconditioned\%20offi ce\%2obuildings\%3A\%2ofindings\%20from\%20a\%20summer\%2osurvey\%2oand\%2ofield\%2oexperiments\% 20in\%20Iran\&author=N.\%20Nazanin\&author=I.\%20Knight\&author=P.\%20Jones\&journal=Indoor\%20Bui lt\%20Environ.\&volume=17\&pages=69-79\&publication_year=2008)

O'Farrell, M., Wild, S., Sabir, B.B.: Pore size distribution and compressive strength of waste clay brick mortar. Cem. Concr. Compos. 23, 81-91 (2001)

CrossRef (https://doi.org/10.1016/So958-9465(00)00070-6)

Google Scholar (http://scholar.google.com/scholar_lookup?

title=Pore\%20size\%20distribution\%20and\%20compressive\%2ostrength\%20of\%2owaste\%2oclay\%2obrick \%20mortar\&author=M.\%20O\%E2\%80\%99Farrell\&author=S.\%20Wild\&author=BB.\%20Sabir\&journal=Ce m.\%20Concr.\%20Compos.\&volume=23\&pages=81-91\&publication_year=2001)

Pontikes, Y., Machiels, L., Onisei, S., Pandelaers, L., Geysen, D., Jones, P.T., Blanpain, B.: Slags with high Al and Fe content as precursors for inorganic polymers. Appl. Clay Sci. 73, 93-102 (2013)

CrossRef (https://doi.org/10.1016/j.clay.2012.09.020)

Google Scholar (http://scholar.google.com/scholar_lookup?

title=Slags\%20 with\%2ohigh\%20Al\%20and\%20Fe\%20content\%20as\%2oprecursors\%2ofor\%2oinorganic\% 2opolymers\&author=Y.\%20Pontikes\&author=L.\%20Machiels\&author=S.\%20Onisei\&author=L.\%20Pandel aers\&author=D.\%20Geysen\&author=PT.\%20Jones\&author=B.\%20Blanpain\&journal=Appl.\%20Clay\%20Sc i.\&volume=73\&pages=93-102\&publication_year=2013)

Rubner, K., Hoffmann, D.: Characterization of mineral building materials by mercury-intrusion porosimetry. Part. Part. Syst. Charact. 23, 20-28 (2006)

CrossRef (https://doi.org/10.1002/ppsc.200601008)

Google Scholar (http://scholar.google.com/scholar_lookup?

title=Characterization\%20of\%2omineral\%2obuilding\%2omaterials\%2oby\%2omercury-

intrusion\%2oporosimetry\&author=K.\%20Rubner\&author=D.\%20Hoffmann\&journal=Part.\%20Part.\%20Sy st.\%20Charact.\&volume=23\&pages=20-28\&publication_year=2006)

Zhang, Z., Provis, J.L., Reid, A., Wang, H.: Fly ash-based geopolymers: the relationship between composition, pore structure and efflorescence. Cem. Concr. Res. 64, 30-41 (2014)

CrossRef (https://doi.org/10.1016/j.cemconres.2014.06.004)

Google Scholar (http://scholar.google.com/scholar_lookup?title=Fly\%20ash-

based\%2ogeopolymers\%3A\%20the\%2orelationship\%2obetween\%20composition\%2C\%2opore\%2ostructur e\%20and\%20efflorescence\&author=Z.\%20Zhang\&author=JL.\%20Provis\&author=A.\%20Reid\&author=H.\% 2oWang\&journal=Cem.\%20Concr.\%20Res.\&volume=64\&pages=30-41\&publication_year=2014）

\section{Copyright information}


(C) Springer Science+Business Media B.V., part of Springer Nature 2017

\author{
About this article \\ Cite this article as: \\ Kamseu, E., Mohamed, H., Sofack, J.C. et al. Transp Porous Med (2018) 122: 77. https://doi.org/10.1007/s11242-017-0990-1 \\ - Received 27 November 2017 \\ - Accepted 12 December 2017 \\ - First Online 28 December 2017 \\ - DOI https://doi.org/10.1007/s11242-017-0990-1 \\ - Publisher Name Springer Netherlands \\ - Print ISSN 0169-3913 \\ - Online ISSN 1573-1634 \\ - $\underline{\text { About this journal }}$ \\ - Reprints and Permissions
}

\title{
Personalised recommendations
}

1. Enhanced extraction of phenolic compounds using choline chloride based deep eutectic solvents from Juglans regia L

Vieira, Vanessa... Ferreira, Olga

Industrial Crops and Products (2018)

2. Lightweight geopolymer composites as structural elements with improved insulation capacity Kakali, Glikeria... Tsivilis, Sotiris

MATEC Web of Conferences (2018)

3. Lightweight geopolymer composites as structural elements with improved insulation capacity Kakali, Glikeria... Tsivilis, Sotiris

MATEC Web of Conferences (2018)

Want recommendations via email? Sign up now Powered by: Recommended ${ }^{\mathbf{R}}$

\section{SPRINGER NATURE}

(C) 2018 Springer Nature Switzerland AG. Part of Springer Nature.

Not logged in University of Modena and Reggio Emilia (3000144974) - CRUI-CARE Italy (3000155420) - CARE 2009 \& 2010 (3000180852) Care Nature (3003532199) 155.185.51.103 